\title{
Gestión de conocimiento: Un modelo para impulsar la investigación en los postgrados
}

\author{
Yánez, Janett* \\ Yánez, Raiza**
}

\section{Resumen}

El presente artículo tiene como objetivo analizar la gestión del postgrado en ciencias administrativas, mención gerencia general con la finalidad de proponer un modelo de gestión del conocimiento (GC) para los estudios de postgrado, tomando como caso de estudio la Maestría en Ciencias Administrativas Mención Gerencia General, Universidad de Oriente, Núcleo Anzoátegui. Se pretende fortalecer e integrar las funciones de docencia e investigación, así como, incrementar la producción científica con mayor pertinencia social. La investigación realizada es de tipo cualicuantitativa, diagnóstica y de campo, se asume la revisión documental y entrevistas en profundidad. Los resultados evidenciaron deficiencias relacionadas con la GC y un débil vínculo con el área de investigación. Se concluye que es necesario reorientar las políticas, planes y objetivos en materia de investigación y GC; utilizar un modelo de GC para redimensionar y fortalecer la investigación; diversificar las líneas de investigación para alinear y articular los esfuerzos investigativos y dar respuesta a las necesidades nacionales, locales y globales; establecer alianzas intra e interinstitucionales; disponer de recursos tecnológicos adecuados, todo ello para impulsar la producción, difusión y transferencia de conocimientos.

Palabras clave: Gestión del conocimiento, investigación, postgrado, universidad.

\section{Recibido: 11-07-11. Aceptado: 31-10-12}

* Maestría en Ciencias Administrativa Mención Gerencia General, Escuela de Ciencias Administrativas. Universidad de Oriente, Núcleo de Anzoátegui.

** Programa Sistemas Gestión de la Calidad, Escuela de Ingeniería y Cs. Aplicados, Universidad de Oriente, Núcleo de Anzoátegui, e-mail: yanezj@udo.edu.ve 


\title{
Knowledge Management: A Model for Encouraging Research among Graduate Students
}

\begin{abstract}
The objective of this article is to analyze the management of graduate studies in administrative sciences in the general management area in order to propose a knowledge management (KM) model for graduate studies, taking as a case the Master's program in Administrative Sciences, General Management, at the University of the East, Anzoategui Campus. The study intends to strengthen and integrate the functions of teaching and research as well as increase scientific production with greater social relevance. The diagnostic, field research is of the quali-quantitative type, using documentary review and in-depth interviews. Results show deficiencies related to KM and a weak link in the research area. Conclusions are that it is necessary to re-orient policies, plans and objectives in matters of research and KM; utilize a KM model to remodel and strengthen research; diversify research lines to align and connect the research efforts and respond to national, local and global needs; establish intra and interinstitutional alliances; and have adequate technological resources available to drive knowledge production, dissemination and transfer.
\end{abstract}

Keywords: knowledge management, research, graduate, university.

\section{Introducción}

La demanda y oferta de los estudios de postgrados, en los últimos años, ha tenido un incremento acelerado en nuestro país, entre otras razones, como consecuencia de una sociedad cada día más exigente y un campo laboral muy competitivo que obliga a los profesionales a realizar estudios de cuarto y quinto nivel (Morlés, 2004).

No obstante, sería simplista considerar sólo el aspecto cuantitativo como elemento de interés en el área de postgrado, en este sentido la creciente reflexión del rol de las universidades como productoras de conocimiento y la responsabilidad social de la investigación, es un factor determinante para ampliar horizontes. Como lo expresa Gibbons (1998), la problemática se orienta a cómo las universidades podrán cumplir con una fun- ción más amplia de producción de conocimiento, más allá de las soluciones locales de problemas centradas en las prioridades académicas.

Hay que añadir que la universidad, como centro generador de conocimientos, y en el caso específico los estudios de postgrados, tienen un papel de suma importancia en la producción y difusión de conocimientos, tal como se planteó en la Conferencia Mundial Sobre Educación Superior de la UNESCO (1998 y 2009), donde se consideró que "la educación superior debería asumir el liderazgo social en materia de creación de conocimientos de alcance mundial para abordar retos mundiales..." (UNESCO, 2009). Desde esta perspectiva, la docencia y la investigación que se realiza en los estudios de postgrados de las universidades venezolanas, tienen el reto de redimensionarse de forma tal, que le permitan adaptar, los 
procesos que en ella se ejecutan a la dinámica social, partiendo del hecho que los conocimientos generados sean pertinentes y sean difundidos y transferidos.

En el caso de Venezuela, es importante resaltar, que los lineamientos y normativas vigentes en el área de postgrado a nivel nacional, enfatizan el vínculo que debe existir entre postgrado e investigación; asimismo, se asume que es evidente que ninguno puede existir sin el otro. Sin embargo, considerando la complejidad del sistema de educación universitaria venezolana, la brecha entre la teoría y la práctica es motivo de preocupación y reflexión; por lo que, los objetivos planteados se encuentran en un terreno álgido a estudiar, tal como se puede interpretar de los resultados obtenidos en el estudio de las reformas universitarias (Fergusson, 2003), en el cual se señala que de 23 experiencias y propuestas de cambios a nivel nacional, sólo ocho (8) corresponden a las universidades autónomas, y ninguna consideró la investigación como un espacio neurálgico a mejorar.

En este orden de ideas, es importante destacar a Ponce (2004), al indicar que no todos los postgrados han sido capaces de adaptarse a la dinámica de cambios de nuestra época en cuanto a la producción de conocimiento pertinentes; por ello señala una serie de debilidades en el ámbito de los postgrados y la investigación, carencia de políticas de investigación, dificultad en la definición/conformación de líneas de investigación, baja tasa de egresados, dificultades organizativas y de gestión que se traducen en no poder dar respuesta a la compleja red de problemas sociales a nivel global y local.
Ante esta situación, se tomó como caso de estudio el Postgrado en Ciencias Administrativas de la Universidad de Oriente, Núcleo de Anzoátegui, planteándose cómo éste puede responder al compromiso de incrementar sus productos de investigación y contribuir al desarrollo de nuestro país; ello se consideró la incorporación de la Gestión del Conocimiento (GC), mediante un modelo teórico, con la finalidad de fortalecer e integrar las actividades docentes y de investigación, en cuanto a la generación, difusión y transferencia de conocimientos, para alcanzar mayor vinculación y pertinencia entre los diversos requerimientos de la institución y la sociedad.

En función de este objetivo, se realizó una investigación tipo cuali-cuantitativa, diagnóstica y de campo, para lo cual se utilizaron técnicas de revisión documental y entrevistas en profundidad a informantes clave.

\section{Investigación, gestión del conocimiento y postgrado}

Diversos autores han hecho hincapié en la necesidad de darle a la producción de conocimientos mayor relevancia, entre ellos Drucker (2002:191), señalando que "el aporte más importante que la gerencia necesita hacer en el siglo XXI es, análogamente, elevar la productividad del trabajo del conocimiento y de quien trabaja con él", es decir, la producción intelectual ha tomado un lugar protagónico en el ámbito organizacional.

De allí que se oriente la atención a las universidades, tradicionalmente las mayores generadoras de conocimientos, ya que tienen el reto de asumir y adaptar- 
se al ritmo acelerado de cambios científicos, tecnológicos y académicos. Rincón y Briceño (2008:2), señalan al citar a Schavino (2002), que dichos cambios obligan a las universidades a dejar atrás "la vieja imagen del claustro universitario cerrada al contexto social...", con la finalidad de dar respuesta a los requerimientos de la sociedad. Es decir, se presentan actualmente brechas y contradicciones entre la teoría y la práctica particularmente en los estudios de postgrados, pues el énfasis de su gestión está basado principalmente en los indicadores del número de egresados y matrícula, y de manera marginal en su productividad científica y la pertinencia de sus actividades docentes y/o de investigación.

De esta manera, para Ruiz (2007: 167), la investigación representa una herramienta fundamental para cumplir con uno de los propósitos prioritarios asignados a las universidades: "crear nuevos conocimientos que serán enseñados, divulgados o utilizados en la resolución de problemas de las propias instituciones o de otros sectores de la sociedad". Es por ello que se concibe la investigación, como la base de la creación de conocimientos en los diferentes campos del saber, y es a través de ésta que las universidades y los estudios de postgrados tienen la oportunidad de ofrecer respuestas a los requerimientos internos y a los de su entorno; al respecto Morín (2007) considera, entre otros aspectos, las necesidades socioeconómicas, el desarrollo tecnocientífico y la protección ambiental en función de la población actual y las generaciones futuras.

Frente al panorama descrito, los estudios de postgrados o llamados por al- gunos autores, estudios de alto nivel, deben plantearse, entre sus objetivos prioritarios, incrementar la generación, difusión y transferencia de conocimientos a través de metodologías y tecnologías innovadoras.

En este sentido, durante las últimas décadas en el marco de la globalización y las tecnologías de comunicación e información, la sociedad en general ha sufrido cambios sustanciales en relación a la gerencia de sus organizaciones e instituciones, originándose un nuevo paradigma, denominado por Rincón y Briceño (2008: 2) denominan, "sociedad de la información / sociedad del conocimiento". En este sentido, es cada vez mayor el número de publicaciones, investigaciones, autores y organizaciones nacionales e internacionales que presentan argumentos, que asumen como base del éxito organizacional el conocimiento y la capacidad de aprendizaje, sin embargo, el conocimiento por sí mismo no es garantía de mejores resultados, de allí la importancia de su gestión, por lo que ha surgido un escenario diferente denominado la gestión del conocimiento.

En este ámbito Yánez y Zavarce (2011), indican que se ha generado una nutrida y creciente diversidad de perspectivas y taxonomías para la clasificación de modelos, fase y conceptualización de la GC que escapan al objetivo de esta investigación, por lo que sin pretender ser exhaustivos, se utilizó como referencia conceptual inicial, la siguiente noción de GC "Proceso de identificar, agrupar, ordenar y compartir continuamente conocimiento de todo tipo para satisfacer necesidades presentes y futuras, para identificar y explotar recursos de conoci- 
mientos, tanto existentes, como adquiridos y desarrollar nuevas oportunidades" (Velásquez, 2007:4), es decir desde una perspectiva que transcienda el corto plazo, los enfoques parcializados o disciplinarios y en función del desarrollo sustentable.

Ahora bien, en cuanto a la GC en el ámbito universitario, la Universidad Autónoma de Madrid, ha marcado pauta con el proyecto de investigación "Gestión del Conocimiento en Universidades y Organismo Públicos de Investigación" (Bueno et al., 2003), el cual tuvo como meta proponer un modelo, basado en el Capital Intelectual $(\mathrm{Cl})$, definido por este equipo como el conjunto de conocimientos que crea o puede crear en el futuro valor para la organización (Diagrama 1).

Por otro lado, de manera complementaria al eje temático desarrollado, para la mejor comprensión de las diversas actividades ejecutadas en los estudios de postgrados, se tomó como referencia a Coelho et al. (2005), quienes utilizan, lo e denominado, el mapa orgánico funcional de los procesos de postgrado, siendo estos una representación del conjunto de las actividades y tareas que se cumplen para satisfacer la demanda de los clientes, es decir, quienes necesitan realizar estudios de cuarto nivel (Diagrama 2), señalando como único producto obtenido, el personal profesional egresado del postgrado.

No obstante, es importante destacar que la universidad como principal centro generador de conocimientos, de acuerdo a las estadísticas oficiales en esta materia, del Observatorio Nacional de Ciencia, Tecnología e Innovación (ONCTI, 2009), no sólo está llamada a re-

\section{Diagrama 1 \\ Modelo gestión del conocimiento en universidades y organismos públicos de investigación}

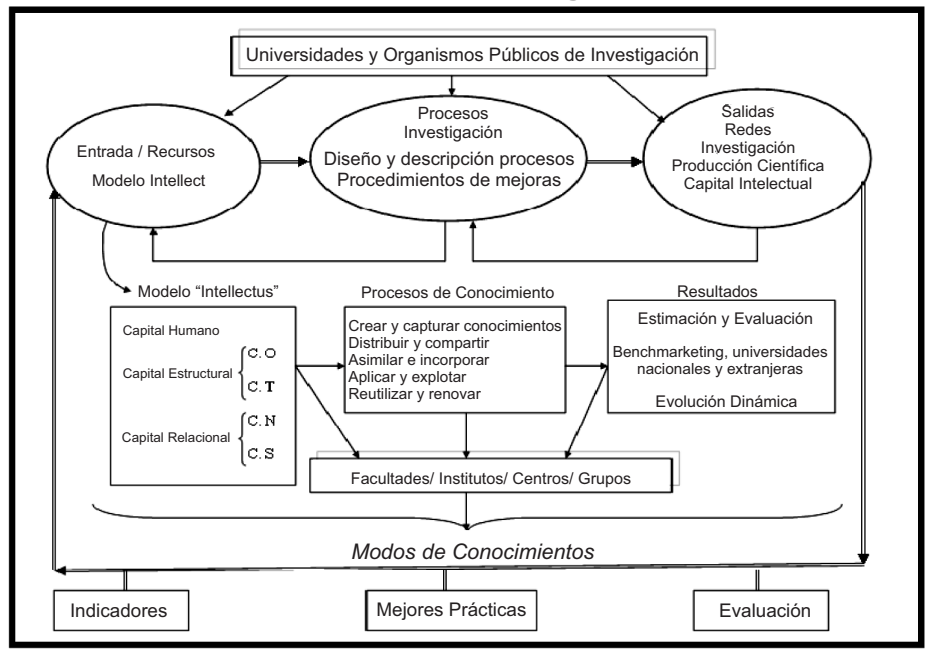

Fuente: Elaboración propia, adaptado de Bueno et al. (2003) y Yánez (2010). 


\section{Sistema de desempeño total de los postgrados}

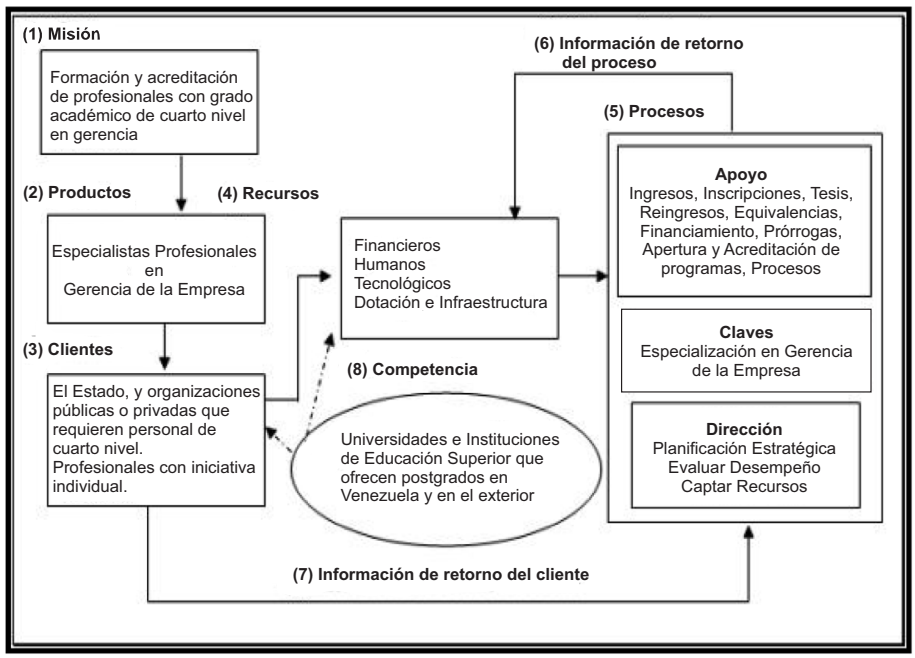

Fuente: Coelho et al. (2005).

visar y redimensionar sus procesos académicos y de investigación, sino debe prepararse para liderizarlos, para lo cual se requieren, cambios profundos en sus estructuras que permitan la compresión de la complejidad actual, como característica inherente de los contextos y objetos de estudio (Yánez y Zavarce, 2011).

\section{Diagnóstico Organizacional: Caso postgrado de ciencias administrativas}

Para realizar el diagnóstico de la situación actual del postgrado en Ciencias Administrativas, mención Gerencia General, de la Universidad de Oriente, Núcleo de Anzoátegui, se utilizó la técnica de la revisión documental, basada en la investigación bibliográfica de artículos científicos de autores nacionales e internacionales, proyectos de investigación, trabajos de grados, informes de gestión, memoria y cuentas, leyes, reglamentos, publicaciones en medios de comunicación públicos e institucionales, entre otros, así como también se aplicó entrevista en profundidad a informantes clave. En primera instancia, a la Coordinadora General de Postgrados de la Universidad de Oriente (Castañeda, entrevista 2010), por ser quien emite las pautas a desarrollar en los programas de postgrados en los diferentes núcleos, obteniéndose como primordial referencia el $\mathrm{Re}$ glamento de los Estudios de Postgrado de la Universidad de Oriente (1997). Posteriormente, se procedió a entrevistar al Coordinador de la Comisión de Investigación (Cáceres, entrevista 2010), el Coordinador del Postgrado de Ciencias Administrativas (Gutiérrez, entrevista 2010), y 
el Delegado de Teleinformática (Guapache, entrevista 2010), todos ellos adscritos al Núcleo de Anzoátegui, con la finalidad de profundizar aspectos que requerían un mayor nivel de información al suministrado en las fuentes documentales consultadas.

Los hallazgos obtenidos fueron complementados y contrastados, tomando como referencia para su recopilación y procesamiento los componentes del Modelo de GC de Bueno et al. (2003), presentado en el Diagrama 1.

El análisis de los resultados obtenidos, muestran una serie de debilidades relacionadas con la GC (Cuadro 1), entre ellos: bajo porcentaje $(13,89 \%)$ de docentes que son investigadores activos y/o están reconocidos por el Observatorio Nacional de Ciencia, Tecnología e Innovación (ONCTI) en el Programa de Promoción al Investigador (PPI), principal referencia en materia de investigación a nivel nacional hasta el año 2011, fecha en la cual el Observatorio nacional de ciencia tecnología e innovación (ONCTI) sustituye el PPI por el Programa de estímulo a la investigación e innovación (PEII) asimismo la investigación no es prioritaria en el postgrado, se realiza primordialmente

\section{Cuadro 1}

\section{Situación de la Maestría en Ciencias Administrativas,} desde la perspectiva de la GC

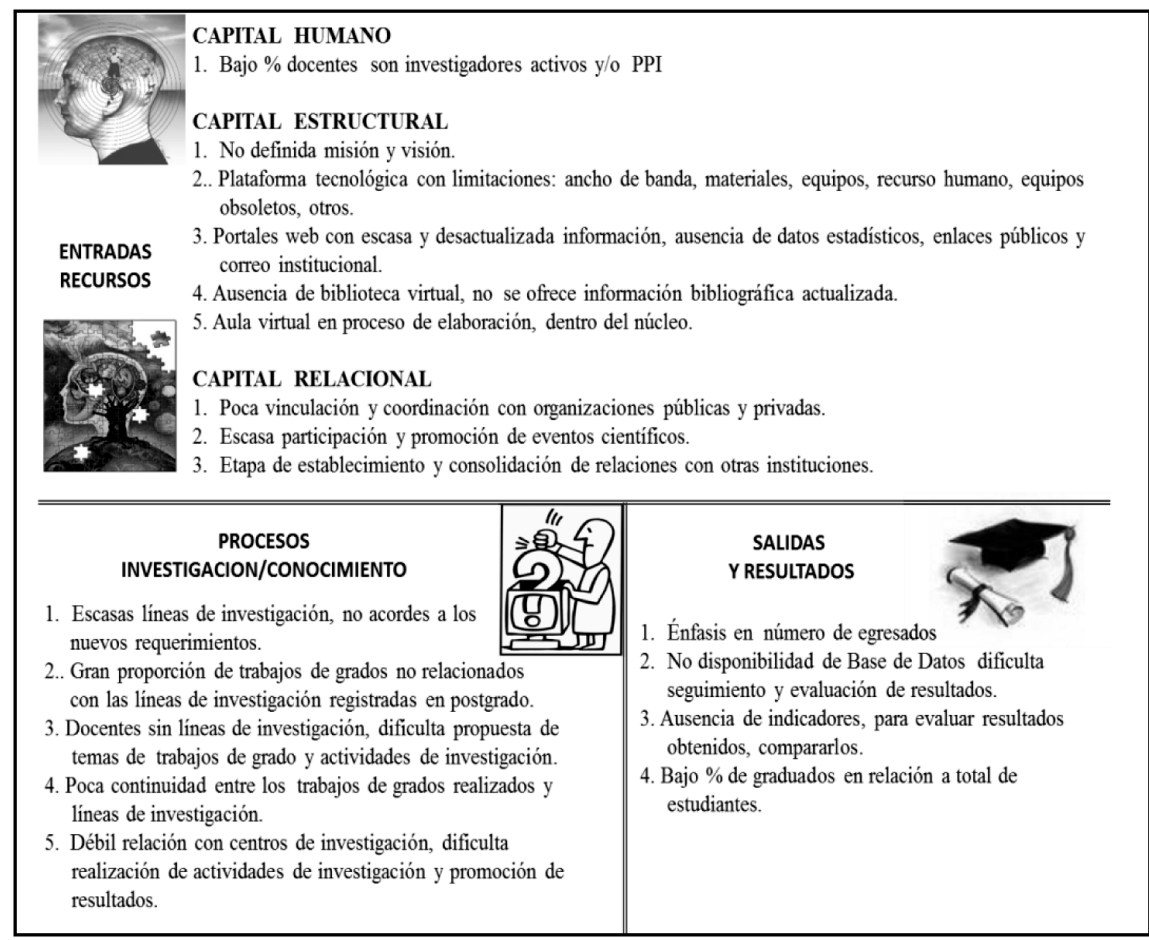

Fuente: Elaboración propia (2010). 
como una actividad que deben cumplir los participantes, para elaborar los trabajos de grado, según la normativa vigente, con la finalidad obtener el título otorgado en el programa de maestría, no como una actividad metódica y disciplinada orientada a dar aportes y soluciones a los principales problemas de la sociedad y/o de las organizaciones, por lo cual la difusión/divulgación de los conocimientos generados y continuidad de los trabajos realizados es muy ocasional y exigua.

Por otra parte, la falta de definición de la misión y visión del programa dificulta la orientación y coordinación de los planes y actividades en función de temas o áreas de interés previamente definidos; limitaciones en el acceso de tecnologías para la GC, basados en el ancho de banda, equipos, materiales e insumos y capital humano que restringe el uso de herramientas básicas como la intranet, internet, video conferencias, entre otros; así como, carencia de un sistemas de información para facilitar el acceso, almacenamiento y difusión de los productos generados (trabajos de grados), artículos científicos, trabajos elaborados en las materias, entre otros. Todo lo anterior se encuentra aunado a un portal web con escasa, desactualizada y/o ausencia de información con relación a datos estadísticos de los resultados obtenidos en las actividades académicas y/o de investigación; así como, ausencia de una biblioteca, aulas virtuales y correos institucionales (Guapache, entrevista 2010). Adicionalmente se tiene poca vinculación con las organizaciones públicas y privadas; a pesar de encontrarse en etapa de establecimiento y consolidación de las relaciones con otras instituciones, estos no se han materializado. Asimismo existe escasa participación y promoción de eventos científicos.

De igual forma, se pudo constatar la existencia de cuatro (04) líneas de investigación, y una gran proporción de trabajos de grados que no se encuentran insertos en las líneas definidas, así como docentes adscritos al programa sin encontrarse insertos igualmente en líneas de investigación, lo que limita las propuestas de temas de trabajos de grado y actividades de investigación; esto aunado a la poca relación de los trabajos de grados realizados con las líneas de investigación y la falta de continuidad de las mismas.

Según Cáceres (Entrevista, 2010) existe una débil relación del postgrado con el Centro de Investigación de Ciencias Administrativas y Económicas (CICAE) lo que significa que se está desaprovechando la oportunidad de participar en actividades de investigación, así como también de la difusión de resultados.

Con relación a la evaluación de los resultados obtenidos no se dispone de una base de datos ni de indicadores, estos se basan primordialmente en el número de participantes por cohortes y egresados por año, presentándose en el período 2006-2008, bajo porcentaje $(4,67 \%)$ de graduados en relación al total de los estudiantes. No obstante, destacan como aspectos positivos, los planes iniciados por la coordinación del postgrado en el 2010 para fortalecer las actividades de docencia e investigación (Gutiérrez, entrevista 2010), entre ellos inicio de las actividades de la revista arbitrada del postgrado, los contactos realizados para establecer acuerdos y convenios con 
otras universidades y centros de investigación, entre otros.

Bueno et al. (2003), señala como uno de los principales obstáculos de la $\mathrm{GC}$ en las universidades y centros de investigación públicos, la ausencia de indicadores, que permitan la evaluación de los resultados y la formulación de acciones de mejora, así como también la escasa difusión/divulgación de los conocimientos generados en las universidades españolas estudiadas en su proyecto, lo que origina que ocupen un "discreto" lugar en lo que se refiere a la producción científica, aspectos que coinciden con los resultados obtenidos en este trabajo.
No obstante, a diferencia de Bueno et al. (2003), se incluyó en esta investigación el Capital Estructural y Relacional por considerarlos necesarios, con mayor o igual importancia que los otros componentes, pues mejoran e incrementan la GC y la producción científicas en las organizaciones, y no sólo como elementos facilitadores.

\section{Modelo de GC para estudios de postgrados}

Con base a lo anteriormente presentado, se desarrolló el "modelo de GC para estudios de postgrados" que se muestra en el Diagrama 3, el cual tiene como objetivo

\section{Diagrama 3 \\ Modelo de GC para Estudios de Postgrados}

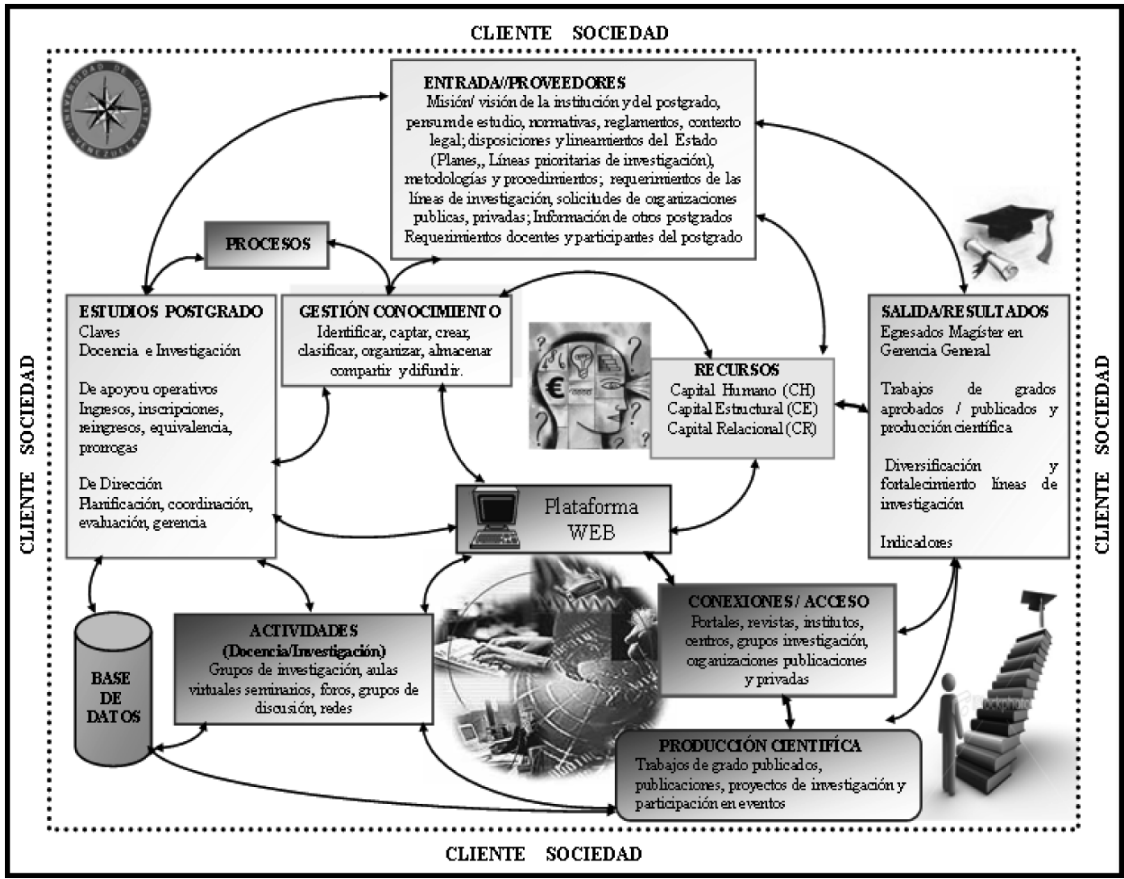

Fuente: Elaboración propia (2010). 
primordial propiciar el fortalecimiento de las actividades académicas e impulsar la investigación, con la finalidad de mejorar los resultados obtenidos, de manera de, no sólo considerar como resultado los trabajos de grado presentados y por ende, los egresados, sino también los proyectos de investigación, artículos científicos y organización/participación en eventos, que le permita una mayor proyección en la comunidad universitaria y en la sociedad en general. Esto implica, la revisión y mejoras de sus procesos (clave, de apoyo y dirección), de naturaleza docente, tecnológicas y administrativas, asociados a la captación, generación y divulgación del conocimiento.

El modelo de GC para estudios de postgrados, se caracteriza porque identifica, crea, clasifica, organiza, almacena y difunde el conocimiento a través de sus componentes que se interrelacionan entre sí, caracterizándose por ser cíclico, continuo, flexible y evolutivo.

El modelo propuesto destaca como punto de partida a los clientes (Estado venezolano, organizaciones públicas/privadas y la sociedad en general), los cuales le suministran entradas al programa, donde a través de los procesos clave (académicos/investigación), organizados y articulados con los procesos de apoyo y dirección, logran la producción académica y la difusión de conocimientos, obteniéndose como resultado: la formación de profesionales, trabajos de grado, diversificación y fortalecimiento de las líneas de investigación, siendo los resultados evaluados a través de indicadores; y a su vez orientados a los clientes, quienes son parte interesada, directa e indirectamente de las actividades de docencia e investigación, a través del impacto y/o contribución que puedan proporcionar los mismos.

Con todo ello, lo que se pretende es hacer énfasis en la interrelación de las actividades de docencia e investigación en función de la GC, con la finalidad de incrementar la producción científica.

Del modelo propuesto, es necesario destacar la importancia fundamental del capital humano, estructural y relacional. En lo que respecta al capital humano, se encuentran los docentes e investigadores, el capital estructural incluye el capital organizacional (misión y visión), el capital tecnológico (internet, sistemas multimedias, workflow, datawarehouse, motores de búsqueda, gestión documental, intranets, Groupware, entre otros) y, el capital relacional conformado por las relaciones entre el programa y las instituciones públicas y privadas, universidades, otros programas de postgrados, centros e institutos de investigación (capital de negocio), así como, la organización y participación en eventos científicos. Los aspectos antes expuestos representan las componentes principales del modelo, los cuales se describen en detalle a continuación:

- Entradas / Proveedores: están constituidas por la misión y visión, tanto de la institución como de los postgrados, el pensum de estudios, normativas y disposiciones legales (Normativa General de Estudios de Postgrados) y reglamentos (tanto internos como externos), así como, las disposiciones y lineamientos establecidos por el Estado en las áreas prioritarias para el desarrollo de la nación (Plan de Desarrollo 
Económico y Social de la Nación (2007-2013), (Chávez, 2007) y, el Plan Nacional de Ciencia, Tecnología e Innovación (2005-2030) (MPPCT, 2005). Así mismo, este componente engloba aquellos requerimientos y sugerencias de los docentes y participantes del postgrado, para la creación de líneas de investigación en áreas de conocimientos de interés en el programa, entre otras.

Por último, en relación a los proveedores, están representados por la propia universidad, el Estado venezolano, y las organizaciones públicas y privadas, y otros programas de postgrados que le suministran insumos al programa para la realización de sus procesos.

- Recursos: de acuerdo a los elementos establecidos del capital intelectual del Modelo Intelect, referido en el Modelo de Bueno et al. (2003), se consideró:

- Capital humano: a través de este componente se estima reforzar las competencias de la investigación de los docentes, así como incrementar la cantidad de docentes investigadores adscritos al programa. Adicionalmente a ello, se considera acrecentar la vinculación entre las líneas de investigación y los trabajos de grado elaborados por los participantes del programa, con la finalidad de dar respuesta a los objetivos prioritarios tanto internos como de su entorno (sociedad), y a su vez, orientar y planificar las actividades académicas desarrolladas en el pensum de estudio del postgrado, en función a ellas, propiciando la generación y transmisión de conocimientos, entre todos los componentes del modelo.
Con el fin de facilitar las actividades de docencia/investigación de los docentes y los participantes del postgrado, se consideró poner a su disposición un espacio en el portal web, propuesto para el programa, que le sirva de herramienta con la finalidad de contribuir a la generación y transmisión de conocimientos, a través de información relacionada con publicaciones científicas, enlaces con organizaciones públicas y privadas, información académica, entre otros. Es importante recordar, que no solo es suficiente la parte tecnológica sino también, que el talento humano, tanto a nivel individual como grupal, participe y se involucre en actividades de aprendizaje y enseñanza, pues es una forma nueva de socializar el conocimiento.

- Capital estructural / Capital organizacional: se refiere a la definición por consenso de la visión, misión, objetivos, metas, estrategias y planes de acción del programa, en los cuales se establezca qué se quiere lograr, conjuntamente con la difusión y puesta en práctica de los mismos. Además, se incluye en este grupo las revistas científicas, que permitan la difusión y divulgación del conocimiento, generado a través de los procesos claves (académicos e investigación), y la producción científica, las publicaciones de trabajos de grado, proyectos de investigación y participación en eventos científicos.

- Capital estructural / Capital tecnológico: se fundamenta en la incorporación de la GC, mediante el uso intensivo y masivo de las Tecnologías de la Información y Comunicación (Pérez y Dressler, 2007), para lo cual se requie- 
re del fortalecimiento de la infraestructura y la tecnología telemática para mejorar la disponibilidad, acceso y calidad de la plataforma web del programa, para sus actividades (docencia/investigación), a través del uso de las diversos medios/recursos, entre los cuales se encuentran, sin ser exhaustivo, internet, mapas de conocimiento, intranet, sistemas multimedias, workflow, datawarehouse, motores de búsqueda, gestión documental, intranets, groupware, blogs entre otros, con la finalidad de disponer de mayor soporte para el almacenamiento, disponibilidad, acceso a la información y difusión del conocimiento, propiciar la creación de grupos de investigación, organización y participación en eventos científicos, así como, la creación de aulas virtuales, redes temáticas y comunidades de prácticas.

Adicionalmente, esto permitirá fortalecer las conexiones/accesos del programa con otros grupos de investigación e instituciones públicas y privadas, mediante el uso de la web. En este sentido, desde el punto de vista académico/investigativo, es importante considerar las redes de aprendizajes, en las cuales se establecen vínculos y articulaciones entre quienes desean aprender (participantes y público en general), lo que representa una valiosa oportunidad para los postgrados, al romper fronteras a través del intercambio de información con actores internos, externos y la sociedad en general y abrirse al trabajo transdiciplinario, intra e interinstitucional.

Mención especial merece, la creación de la base de datos de los postgra- dos, donde se incluya los datos de las actividades y productos de los procesos que se realizan, permitiendo la planificación, análisis, evaluación y control de los resultados, proyectos de investigación, artículos publicados, trabajos de grado en curso, presentados, publicados, metodologías, procedimientos, cursos, informes de gestión, eventos, entre otros.

- Capital relacional/Capital de negocio: comprende la coordinación de las relaciones sistematizadas entre los postgrados y las organizaciones públicas y privadas, universidades y otros programas de otras instituciones de educación superior, considerándose además, las relaciones con la sociedad en general.

- Capital relacional/Capital social: se incluyen las actividades de promoción y difusión que ejecutan los integrantes de los postgrados en eventos científicos y/o actividades de fomento en la generación y divulgación de conocimiento en espacios sociales, que vinculan las actividades docente/investigación en un contexto local, nacional y global, con la incorporación en el programa de redes con otras universidades nacionales e internacionales $y$ grupos de investigación.

Procesos (estudios de postgrados y GC): en lo que concierne a los procesos de los estudios de postgrados que se podrían denominar habituales o tradicionales. Se toma como base a Valarino y Yaber (2001), quienes los clasifica en: clave, de apoyo y dirección, haciéndose énfasis de acuerdo a los objetivos de este trabajo en los procesos clave (académicos y de dirección). 
En este sentido, en función de la investigación realizada y los resultados obtenidos, se conceptualiza la GC en los estudios de postgrado como el conjunto de procesos vinculados a las funciones de docencia e investigación, apoyados en el uso de herramientas tecnológicas para la administración de los capitales humano, estructural y relacional, mediante los cuales se planifica, organiza, coordina, controla y evalúa la creación, captación, adaptación, almacenamiento y difusión, de conocimiento contextualizado en y para la sociedad, con visión prospectiva de acuerdo a las líneas de investigación establecidas.

De esta manera, teniendo como referencia esta definición, los estudios de postgrado pueden revisar, redimensionar y/o definir todos sus procesos, así como sus elementos estratégicos (misión, visión, objetivos y planes), en función de una nueva perspectiva u horizonte que le facilite incrementar su productividad científica y por ende, su calidad académica.

Con respecto al uso de las herramientas web para facilitar las actividades de aprendizaje y socialización del conocimiento, tanto en los procesos clave como de apoyo y dirección, se propone ampliar y diversificar las herramientas informáticas y computacionales en esta materia, como por ejemplo contar con una base de para facilitar el acceso a directorios donde se muestren las principales fuentes de referencia, publicaciones y revistas científicas, organizaciones públicas y privadas vinculadas al área, todo ello con el fin de contar con los mecanismos para que el conocimiento tácito se convierta en conocimiento explicito.
- Salida y resultados: se refiere a los diferentes productos obtenidos en los procesos del postgrado, entre los cuales se encuentran los egresados del programa; los trabajos de grado aprobados, los cuales deben estar vinculación con líneas y/o proyectos de investigación, así como también ser difundidos, en revistas arbitradas, eventos científicos, actividades de difusión en la comunidad universitaria, organizaciones públicas/privadas y comunidades interesadas, generándose a su vez los indicadores de gestión necesarios para la evaluación y control.

En cuanto a las líneas de investigación (fortalecimiento y diversificación), se plantea la conformación de líneas en el marco de los lineamientos del Estado venezolano, vinculadas con las necesidades nacionales, regionales, locales, así como también los desafíos globales, como el desarrollo sustentable, y locales en el ámbito de requerimientos institucionales.

- Clientes/sociedad: uno de los aspectos más resaltantes del modelo es la amplitud en su proyección y el alcance, al considerar como cliente a la sociedad de manera integral, y no sólo a los profesionales interesados o que cursan estudios de postgrado, sino también al Estado venezolano, las organizaciones públicas y privadas, grupos, redes de investigación, comunidades organizadas y público en general, tanto a nivel nacional como internacional.

\section{Conclusiones}

Las conclusiones del presente trabajo apuntan a una serie de desafíos y 
compromisos frecuentes en los estudios de postgrados, entre los cuales destacan el rol en la producción, difusión y transferencia de conocimiento, para dar respuesta a los problemas de la sociedad y a la vez, considerando mayor proyección de sus procesos funcionales, y de esta forma, no sólo circunscribirse a los egresados como los únicos productos obtenidos. En este sentido, se desarrolló un modelo de GC que facilita la identificación, creación, clasificación, organización, almacenamiento y divulgación del conocimiento a través de la interacción cíclica y continua de sus componentes, basado en la necesidad del fortalecimiento e impulso de la investigación con mayor pertinencia y su interrelación con las actividades académicas, aspectos fundamentales en las funciones asignadas a las universidades; en el contexto normativo vigente y escenarios planteados, así como en la dinámica de los procesos de cambios actuales en la denominada sociedad del conocimiento.

Asimismo, en el caso de estudio, es necesario destacar la importancia, de que la organización del postgrado, emprenda esfuerzos cónsonos y consistentes en función de propiciar las condiciones idóneas para la puesta en práctica del modelo de $\mathrm{GC}$, para lo cual se requiere definir la visión, misión y redimensionar las políticas, planes y objetivos del postgrado en función de los requerimientos de la GC, así como también, establecer líneas de investigación, cónsonas con los lineamientos establecidos en esta materia por el Estado venezolano, para orientar y articular los esfuerzos investigativos a la solución de problemas nacionales, locales y/o globa- les, propiciando la continuidad de los trabajos y esfuerzos, y no solo individualidades. Adicionalmente, es preciso establecer vínculos/alianzas con centros investigación y otras organizaciones, con la finalidad de diversificar/fortalecer las líneas de investigación en la perspectiva de trabajos multi y transdisciplinarios, todo ello, para impulsar la producción, promoción, difusión y transferencia de conocimientos, para lo cual es necesario, mas no suficiente, disponer y utilizar los recursos tecnológicos adecuados a los objetivos y planes en materia de GC e investigación que el postgrado se plantee.

\section{Referencias bibliográficas}

Asamblea Nacional de la República Bolivariana de Venezuela (2001). Normativa General de los Estudios de Postgrado para las Universidades e Institutos debidamente autorizados por el Consejo Nacional de Universidades. Aprobada el 06-07-2001 en Consejo Universitario. Disponible: http://legal.com.ve/leyes/C332.pdf. [Consultado: 2010, Enero 2010].

Bueno, Eduardo; Morcillo, Patricio, Rodríguez, Jesús; Luque, María; Cervera, Mercedes; Camacho, Claudia; Merino, Belén; Murcia, Cecilia; Rodríguez, Oscar; Villanueva, Julián y Villar, Lidia (2003). Gestión del Conocimiento en Universidades y Organismos Públicos de Investigación. Proyecto de Investigación de la Universidad Autónoma de Madrid. Disponible en: www.madrimásd.org [Consultado: 2009, Febrero 10].

Coelho, Franklin; Romero, Mariana y Yaber, Guillermo (2005). Indicadores de Desempeño Clave para Programas Académicos de Postgrado. Investi- 
gación y Postgrado. Vol. 20, No.2, p.123-153. Disponible en: http://www. scielo.org.ve/scielo.php?Script=sci_ arttext\&pid=S1316-00872005000200 005\&lng=es\&nrm=iso. [Consultado: 2010, Junio 20].

Drucker, Peter (2002). La Gerencia en la Sociedad Futura. Grupo Editorial Norma. Bogotá.

Fergusson, Alex (2003). Relevamiento de Experiencias de Reformas Universitaria en Venezuela. Informe Final. Proyecto IESALC-UNESCO. Consultado el 15 de Junio 2010. Disponible en: http://www.iesalc.unesco.org.ve/ programas/reformas/venezuela/Informe\%20Reformas \%20Venezuela\% 20-\%20Final.pdf. [Consultado: 2010, Junio 15].

Gibbons, Michael (1998). Pertinencia de la Educación Superior en el Siglo XXI. Association of Commonwealth Universities Washington, D.C. Banco Mundial. Disponible en: http://www. cendesucv.edu.ve/biblioteca_CENDES.jsp [Consultado: 2009, Octubre 15].

Morín, Edgar (2007). Investigación y Formación Docente. Colegio Universitario de Los Teques "Cecilio Acosta". Coordinación de Investigación Recursos Humanos CULTCA.

Observatorio Nacional de Ciencia Tecnología e Innovación (ONCTI) (2009). Observatorio Nacional de Ciencia Tecnología e Innovación. Disponible en: http://onctic.gob.ve/. [Consultado: 2009, Junio 09]

Ministerio del Poder Popular para la Ciencia, Tecnología e Innovación (MPPCT) (2005). Plan Nacional de Ciencia, Tecnología e Innovación 20052030. Ministerio de Ciencia y Tecnología, República Bolivariana de Venezuela. Disponible en: http://www.gobiernoenlinea.ve/misc-view/sharedfiles/Plan-Nacional-CTI.pdf. [Consultado: 2010, Mayo 05]
Chávez Frías, Hugo Rafael (2007). Líneas Generales del Plan de Desarrollo Económico y Social de la Nación 2007. 2013. Disponible: http://www.portaleducativo.edu.ve/Politicas_edu/planes/documentos/Lineas_Generales_ 2007_2013.pdf [Consultado: 2010, Marzo 02].

Pérez, Daniel; Dressler, Matthias (2007). Tecnologías de la información para la gestión del conocimiento. Intangible Capital No 15. Vol. 3. pp. 31-59, Ene-Mar de 2007. Disponible en: http://www.intangiblecapital.org/index.php/ic/article/viewFile/12/18. [Consultado: 2010, Febrero 02].

Ponce, Víctor (2004). Tendencias, perspectivas y retos del posgrado y la investigación en Latinoamérica. Revista Red de Postgrado en Educación. Octubre 2004. Disponible en: http://www. Clacso-posgrados.net/documentos aportes/35.pdf. [Consultado: 2010, Marzo 02].

Ruiz, Carlos (2007). La Universidad Venezolana en una Época de Transición. Profesor Universidad Pedagógica Experimental Libertador-Instituto Pedagógico de Barquisimeto (UPEL-IPB). Disponible en: http://www.ucla.edu. Ve/dac/investigaci\%F3n/compendium7/Epoca\%20de\%20Transicion. htm. [Consultado: 2010, Mayo 10]

Rincón de Parra, Haydee y Briceño, Magaly (2008). Producción y Gerencia del Conocimiento en la Universidad de Ios Andes en Venezuela. Revista de Universidad y Sociedad del Conocimiento. Volumen 5 No 1. p.1-8. Disponible en: http://redalyc.uaemex.mx/ pdf/780/78011203003.pdf. [Consultado: 2010, Abril 25].

Universidad de Oriente (1997). Reglamento de los Estudios de Postgrado de la Universidad de Oriente. Vicerrectorado Académico. Disponible en: http:// vrad.udo.edu.ve/sistemas/index. 
php?option=com_content\&view=article\&id=449 [Consultado: 2009, Mayo 3].

UNESCO (1998). Conferencia Mundial de Educación Superior. La Ciencia y la Cultura: Declaración mundial sobre la educación superior en el Siglo XXI: Visión, acción. Marco de acción prioritaria para el cambio de la educación superior. Conferencia Mundial sobre la Educación Superior. Paris Octubre 1998. Disponible en: http://www.cres 2008.org/upload/documentosPublicos/docs_aportes/Declaracion\% 20ES\%20Paris\%201998.pdf. [Consultado: 2008, Abril 10].

UNESCO (2009). Conferencia Mundial de Educación Superior 2009: Las Nuevas Dinámicas de la Educación Superior y de la Investigación para el Cambio Social y el Desarrollo. París, 5-8 de Julio de 2009. Borrador Final. Disponible en: http://www.unesco.org/ education/hed/2009-worldconference. [Consultado: 2009, Abril 15].

Valarino, Elizabeth y Yaber, Guillermo (2001). Productividad Académica en la Investigación de Postgrado. Agenda Académica. Departamento de Ciencias y Tecnología del Comportamiento Universidad Simón Bolívar. Volumen 8. p.3-14. Disponible: http://www. intercontacto.com/nivel1/PDF/Articulos/20anos.PDF. [Consultado: 2010, Mayo 27]

Velásquez, Laity (2007). Las Redes de Investigación Virtuales: Propuesta de Fomento y Desarrollo de la Cultura In- vestigativa en las Instituciones de Educación Superior. Revista de Universidad y Sociedad del Conocimiento. Volumen 4 No. 2, p 1-11. Disponible: http://www.uoc.edu/rusc/ 4/2/ $\mathrm{dt} / \mathrm{esp} / \mathrm{velasquez}$.pdf. [Consultado: 2010: Mayo 27].

Yánez, Raiza (2010). Desarrollo Sustentable, Gestión del Conocimiento y la Universidad Venezolana. Tesis Doctoral Universidad Yacambú. Enero 2010.

Yánez, Raíza y Zavarce, Carlos (2011). Desarrollo Sustentable, Universidad y Gestión del Conocimiento desde la Perspectiva Luhmaniana. Revista Iberoamericana de Ciencia, Tecnología y Sociedad. $N^{\circ} 17$. Disponible en: http://www.revistacts.net/files/Volumen\%206\%20-\%20N\%C3\%BAmero\%2017/yanez_corregido.pdf [Consultado: 2011, Mayo 2011].

\section{Entrevistas:}

Cáceres, Alfonso. Coordinador de la Comisión de Investigación, Núcleo de Anzoátegui. Barcelona, 08 Junio de 2010.

Castañeda, Haydee. Coordinadora General de Postgrado Universidad de Oriente, Anzoátegui. Barcelona, 18 Mayo de 2010.

Guapache, René. Delegado de Teleinformática Universidad de Oriente, Núcleo de Anzoátegui. Barcelona, 10 Junio de 2010.

Gutiérrez, Lucas. Coordinador del Postgrado de Cs Administrativas Núcleo Anzoátegui. Barcelona, 15 Junio de 2010. 